\title{
METACOGNICIÓN EN UN PROCESO DE APRENDIZAJE AUTÓNOMO Y COOPERATIVO EN EL AULA UNIVERSITARIA
}

\author{
Alicia Pérez de Albéniz Iturriaga \\ Universidad de La Rioja \\ Elena Escolano Pérez \\ Universidad de Zaragoza \\ María Teresa Pascual Sufrate \\ Universidad de La Rioja \\ Beatriz Lucas Molina \\ Universidad de Valencia \\ Sylvia Sastre i Riba \\ Universidad de La Rioja
}

\begin{abstract}
RESUMEN: En el marco del Espacio Europeo de Educación Superior la docencia debe estar guiada y gestionada en función del aprendizaje de los alumnos. Los estudiantes necesitan activar las competencias necesarias para Ilevar a cabo un aprendizaje autónomo. En este contexto, se realizó una experiencia en la asignatura Psicología de la Educación con los estudiantes de primer curso del Grado de Educación Primaria de la Universidad de La Rioja. El objetivo consistió en conocer el análisis metacognitivo que realizaban los alumnos cuando se les presentaba tareas cuya resolución exigía la combinación de estrategias de aprendizaje autónomo y cooperativo. De acuerdo con lo esperado, los estudiantes, tras realizar un análisis metacognitivo acerca de la experiencia y su aprendizaje a través de esta metodología, ofrecen valoraciones positivas. Asimismo, los alumnos afirman haber conseguido un mayor dominio de los contenidos trabajados mediante la elaboración autónoma y cooperativa en comparación con otras técnicas de estudio utilizadas habitualmente, basadas más en un aprendizaje receptivo.
\end{abstract}

PALABRAS CLAVE: Aprendizaje autónomo, aprendizaje cooperativo, metacognición, autoconocimiento, autorregulación.

\section{METACOGNITION IN A PROCESS OF AUTONOMOUS AND COOPERATIVE LEARNING IN THE UNIVERSITY CLASSROOM}

ABSTRACT: In the new framework of the European Higher Education Area, teaching should be guided and managed in light of students learning. Students 
need to activate the necessary competencies to carry out an autonomous learning. In this context, an experience was conducted in the subject Psychology of Education with the first year Pre-service Elementary School Teachers from the University of La Rioja. The objective was to know the metacognitive analysis made by students when they had to do tasks that required both autonomous and cooperative strategies. As expected, results from the metacognitive analysis revealed a positive evaluation of this methodology. Moreover, students reported having achieved a better mastery of contents worked through the autonomous and cooperative development in comparison with those worked through common strategies based on receptive learning.

KEYWORDS: Autonomous learning, cooperative learning, metacognition, self-knowledge, self-regulation.

Recibido: $21 / 03 / 2014$

Aceptado: 20/09/2014

Algunos planteamientos advierten que la sociedad de nuestro tiempo se caracteriza por un gran ritmo de cambio tecnológico y científico (e.g., Mateos y Pérez, 2006; Pozo y Monereo, 2009). Esta situación imposibilita poder prever qué conocimientos específicos tendrán que dominar los actuales estudiantes dentro de diez o quince años para poder afrontar las demandas reclamadas en su ejercicio profesional. Por tanto, la formación universitaria no puede aportar todos los conocimientos y saberes necesarios para un futuro ejercicio profesional. Sin embargo, lo que sí puede y debe hacer es formar a los futuros profesionales para que sean unos aprendices más flexibles, eficaces y autónomos, dotándoles de capacidades de aprendizaje y no sólo de conocimientos o saberes específicos que suelen ser menos duraderos.

En este sentido, la sociedad demanda profesionales capaces de gestionar el conocimiento, es decir, capaces de aprender y de usar de distintas formas el saber acumulado. La universidad española se ha encontrado ante el reto de innovar la manera en la que se enseña y aprende en sus aulas, con el fin de satisfacer la necesidad de aprender a aprender. De hecho, diversos autores han puesto de manifiesto la necesidad de considerar la autonomía del aprendizaje de los estudiantes como un fin último de la enseñanza universitaria (Monereo y Pozo, 2003; Pozo, 2003; Pozo y Mateos, 2009; Pozo y Monereo, 2009). Tal y como plantean De la Fuente, Pichardo, Justicia y Berbén (2008), el estudiante precisa una elevada autonomía e independencia para aprender, regulando su disposición afectivo-motivacional y sus procesos cognitivos.

De hecho, son diversos los modelos que se han desarrollado para analizar las variables que afectan a la calidad de la autorregulación en el proceso de enseñanza-aprendizaje. Como ejemplo de nuestro contexto, se destaca el modelo DIDEPRO (De la Fuente y Justicia, 2007; De la Fuente, Justicia y Berbén, 2005) que adopta las características del modelo 3P (Presagio-Proceso-Producto) de Biggs (2001) y del modelo de Zimmerman y cols. (Zimmerman y Kintasas, 1997). 
Este planteamiento fue especialmente importante en el diseño y puesta en marcha de los planes de estudio siguiendo la filosofía del Espacio Europeo de Educación Superior. Según este planteamiento, la formación universitaria debía dirigirse a la formación de profesionales competentes, capaces de adaptarse y responder a las demandas cambiantes y flexibles de la sociedad y por tanto, del ejercicio profesional.

En este sentido, García-Valcárcel (1994) distingue dos modelos de enseñanza universitaria denominados modelo expositivo y modelo interactivo. Dichos modelos se diferencian por determinadas pautas de actuación docente relacionadas con la interacción y motivación de los estudiantes. Mientras que el modelo expositivo se orienta más al contenido, el modelo interactivo se centra más en el proceso de aprendizaje como, por ejemplo, presentar de manera explícita los objetivos, adaptarse al nivel de conocimientos de los estudiantes, considerar sus intereses y necesidades, relacionar los contenidos de la asignatura con problemas significativos para ellos, ser accesible y cercano a los estudiantes estableciendo continuamente vías de participación, etc.

En la línea del modelo interactivo se sitúan estrategias como el aprendizaje autónomo y el aprendizaje cooperativo. Tal y como señalan Monereo y Pozo (2003), entre las competencias más importantes que deben adquirir tanto profesores como alumnos se encuentran el enseñar/aprender a aprender y pensar y el enseñar/aprender a cooperar.

En concreto, el aprendizaje autónomo es aquél en el que el alumno tiene la facultad de tomar decisiones que le permiten regular su propio proceso de aprendizaje con el fin de aproximarse a una meta, en el seno de unas condiciones específicas que forman el contexto de aprendizaje. Ser autónomos aprendiendo supone dominar un conjunto amplio de estrategias, o lo que es lo mismo, ser capaces de tomar decisiones intencionales, conscientes y contextualizadas con el fin de lograr los objetivos de aprendizaje perseguidos (Monereo y Pozo, 2003). Es preciso remarcar que la autonomía en el aprendizaje se concede con respecto a las condiciones pero no sobre su resultado o producto. El estudiante debe dar respuesta a las demandas específicas de conocimiento formuladas por el profesor, pero escogerá por sí mismo aquellas condiciones contextuales de tiempo, lugar, herramientas, etc. que estime necesarios para elaborar aquella respuesta (Rué, 2009).

El aprendizaje autorregulado exige un proceso activo en que los estudiantes establecen los objetivos que lo guían intentando monitorizar, regular y controlar su cognición, motivación y comportamiento con la intención de alcanzarlos (Rosário, 2004). De ahí la importancia de conocer el proceso metacognitivo subyacente.

En resumen, lograr que los aprendices sean más autónomos, es decir, capaces de autorregular sus acciones para aprender, implica hacerlos más conscientes de las decisiones que toman, de los conocimientos que ponen en juego, de sus dificultades para aprender y del modo de superarlas (Monereo y Pozo, 2003).

Por su parte, el aprendizaje cooperativo es un término genérico usado para referirse a un grupo de procedimientos de enseñanza que parten de la organización de la clase en pequeños grupos mixtos y heterogéneos donde los alumnos trabajan conjuntamente de forma coordinada para resolver tareas académicas y profundizar en su propio aprendizaje. Diversos autores (Johnson y Johnson, 1989; Johnson, 
Johnson y Holubec, 1999) lo definen como aquella situación de aprendizaje en la que los objetivos de los participantes se hallan estrechamente vinculados, de tal manera que cada uno de ellos solo puede alcanzar sus objetivos si, y sólo si, los demás consiguen alcanzar los suyos. El aprendizaje cooperativo se centra así en la interacción alumno-alumno, favoreciendo la regulación entre compañeros. Su eficacia se debe a la confrontación entre perspectivas diferentes, a la vez que fuerza a los participantes a explicitar y redescribir su propia perspectiva para poder integrarla con la de los demás y poder tomar decisiones conjuntas potenciándose una mayor conciencia y control de los propios procesos de aprendizaje en cada de uno de ellos. Al intentar comprender las ideas de los otros y reconciliarlas con las propias, los alumnos se implican en un proceso de negociación de significados que puede conducir a la co-construcción o construcción conjunta de nuevos conocimientos, de nuevos procesos de pensamiento y de estrategias de solución de problemas (Mateos y Pérez, 2006).

Pero las estrategias de aprendizaje autónomo y cooperativo no son excluyentes (Escribano, 1995). Es más, frecuentemente, un incremento de la autonomía va asociado a un aumento de la cooperación. La necesidad de explicitar el propio conocimiento (no sólo en sus productos sino también en sus procesos) para poder hacer un uso autónomo y metacognitivo del mismo, hace especialmente eficaz el diseño de espacios de aprendizaje cooperativo, ya que la explicitación constituye un modo de comunicación y por tanto, se favorece en contextos de interacción (Barkley, Cross y Major, 2005; Pozo y Mateos, 2009).

El aprendizaje autónomo se encuentra estrechamente vinculado a la capacidad de metacognición, definida como la competencia que permite ser conscientes de parte de nuestra cognición, es decir, de algunos de los procesos y productos que elaboramos en nuestra mente (Monereo y Pozo, 2003). El aprendizaje eficaz exige una gestión metacognitiva del conocimiento, es decir, exige el conocimiento y control de las propias actividades y procesos de aprendizaje (De la Fuente y Justicia, 2003; Mateos, 2001; Pozo y Mateos, 2009). La metacognición implica dos aspectos estrechamente relacionados (Weinert y Kluwe, 1987; Schraw y Dennison, 1994), aunque no siempre coincidentes: 1 ) el conocimiento del propio conocimiento, es decir, lo que la persona sabe sobre sus propios procesos y productos de aprendizaje y 2) la regulación o control sobre la propia actividad cognitiva, el uso que hace de su conocimiento y procesos cognitivos mientras está resolviendo una tarea.

La metacognición se compondría, por lo tanto, de un componente de naturaleza declarativa, "saber qué" acerca de la propia actividad cognitiva, y de otro componente de naturaleza procedimental, "saber cómo" aplicar los procesos cognitivos de manera eficaz en el contexto de una determinada tarea. Este componente procedimental debe estar presente en tres momentos distintos de la resolución: antes, durante y después de ella (Pozo y Mateos, 2009), correspondiéndose con tres procesos cognitivos: planificación de la actividad, autorregulación de su ejecución y evaluación de los resultados obtenidos, respectivamente. No obstante, es evidente que el resultado de cada proceso puede convertir la realización de la tarea en un proceso recursivo y no lineal. Por ejemplo, la autorregulación de nuestra ejecución en ocasiones puede exigir una nueva planificación de las estrategias a poner en marcha 
para la resolución de la tarea, sin necesidad de esperar a la obtención, y por tanto evaluación, de unos resultados finales.

Además, es importante señalar que la metacognición (y, por tanto, las competencias que implica) puede mejorarse cuando los alumnos están aprendiendo (De la Fuente y Justicia, 2003; Zimmerman y Kintasas, 1997).

En este contexto, se realizó una experiencia cuyo objetivo fue conocer el análisis metacognitivo de un grupo de alumnos universitarios sobre su propio proceso de aprendizaje durante la resolución de una tarea que requería la combinación de estrategias de aprendizaje autónomo y cooperativo. Esta experiencia, fue llevada a cabo con alumnos del primer curso del Grado de Educación Primaria de la Universidad de La Rioja.

\section{MÉTOdo}

\section{Participantes}

La muestra estuvo formada por los 28 alumnos de la asignatura de Psicología de la Educación, componentes de un Grupo Reducido (clases de prácticas en los que los alumnos trabajan en grupos de reducido tamaño) de dicha asignatura.

\section{Instrumentos}

El material de estudio consistió en un capítulo teórico sobre la "motivación" (Sampascual, 2007).

Se elaboró ad hoc un caso práctico (Anexo 1) en el que se recogían diferentes situaciones reflejo de la falta de motivación en los alumnos de una supuesta clase de cuarto curso de Educación Primaria.

Se adaptó ad hoc (Anexo 2) un cuestionario para conocer los procesos metacognitivos de los alumnos basado, parcialmente, en ítems adaptados de la escala de Regulación del Inventario de Conciencia Metacognitiva (Metacognitive Awareness Inventory, MAI, Schraw y Dennison, 1994). Consiste en cinco preguntas cerradas sobre el resultado del aprendizaje mediante la elaboración autónoma y cooperativa de la tarea, y cinco preguntas cerradas relativas a la regulación de la planificación de las estrategias motivacionales. La respuesta a estas preguntas se realiza mediante una escala Likert de cinco puntos, siendo el 1 el indicador de la valoración Totalmente en desacuerdo y el 5 el indicador de la valoración Totalmente de acuerdo.

De igual manera, incluye dos preguntas abiertas sobre tres ventajas y tres limitaciones de estos tipos de aprendizaje.

\section{Procedimiento}

El presente proyecto se llevó a cabo en la asignatura Psicología de la Educación en el Grado de Educación Primaria de la Universidad de La Rioja. Se trata de una asignatura de carácter obligatorio del primer curso de la titulación, perteneciente al módulo de Formación Básica y se desarrolla a través de 6 créditos ECTS. Esta asignatura se imparte en el primer semestre de primer curso. Está compuesta por 12 unidades temáticas. El presente proyecto de innovación se aplicó en la unidad 9: La motivación. 
Los instrumentos de medida se administraron de manera grupal. Los alumnos fueron informados previamente de la confidencialidad de sus respuestas y de la naturaleza voluntaria de su participación. Los participantes no recibieron ningún incentivo monetario o de otro tipo. Para la recogida de la información se obtuvo el consentimiento informado de los mismos.

El procedimiento seguido se dividió en tres fases. En la primera fase se explicó a los alumnos el plan de trabajo previsto para conocer quiénes aceptaban participar y se comprometían con la tarea. Para ello: a) se planteó un caso práctico (ver Anexo 1) con el doble objetivo de incitar el interés hacia el estudio del tema y la comprensión de la importancia de motivar a sus alumnos; b) se planteó que su participación supondría una facilitación del aprendizaje del tema. En la segunda fase, se concretó el caso práctico a resolver en dos fases de trabajo: a) trabajo individual y, b) trabajo en grupos de tres alumnos. El trabajo individual se basó en el aprendizaje autónomo. Cada alumno del grupo estudió de manera autónoma una de las teorías sobre motivación tratadas en la documentación ofrecida por la profesora y las estrategias motivacionales que se derivan de las mismas. Asimismo, realizó un mapa conceptual de dicha teoría y una propuesta para la resolución del caso. El trabajo en grupos se basó en estrategias de aprendizaje cooperativo, subdividiéndose en dos acciones: 1) cada alumno compartió con los compañeros de su grupo el mapa conceptual realizado en la fase anterior y discutió las discrepancias. Asimismo, cada grupo tuvo que exponer las propuestas individuales consensuando las estrategias más adecuadas para aplicar en el aula en la resolución del caso planteado; 2) tres alumnos, seleccionados al azar y pertenecientes a grupos diferentes, expusieron al resto del grupo la teoría motivacional elaborada, de modo que, todas las teorías fueron expuestas y discutidas. Igualmente, cada situación del caso práctico fue analizada de manera pormenorizada desde cada una de las teorías estudiadas por diferentes alumnos seleccionados al azar.

La tercera fase consistió en el análisis metacognitivo de la actividad. Los alumnos respondieron, individualmente, al cuestionario construido ad hoc (ver Anexo 2). La primera parte del cuestionario tuvo como objetivo la evaluación de los resultados de aprendizaje obtenidos. En la segunda parte del cuestionario se respondió a dos preguntas abiertas acerca de las ventajas e inconvenientes que habían percibido los alumnos con este tipo de aprendizaje. Finalmente, la tercera parte de este cuestionario, basada en ítems adaptados de la escala de Regulación del MAI (Schraw y Dennison, 1994), tuvo el objetivo de evaluar y hacer conscientes para los alumnos las estrategias de regulación metacognitiva que habían utilizado como resultado de su participación en las actividades del tema.

\section{Análisis de datos}

En primer lugar se procedió a la categorización de las respuestas abiertas mediante juicio de expertos y codificación alfanumérica. Posteriormente se realizó un vaciado de las respuestas cerradas. Finalmente se realizaron los análisis descriptivos y cálculo de diferencias significativas mediante el estadístico Chi cuadrado con el programa estadístico SPSS 15.0 (Statistical Package for the Social Sciences, 2006). 


\section{RESULTADOS}

Los resultados se presentan en tres apartados: 1) percepción de los resultados del aprendizaje autónomo y cooperativo, 2) ventajas e inconvenientes del aprendizaje autónomo y cooperativo y 3) regulación de la planificación durante la resolución.

\section{Percepción de resultados del aprendizaje autónomo y cooperativo}

Tal como refleja la Figura 1, los resultados indican que la mayoría de participantes están Totalmente de acuerdo o De acuerdo con las afirmaciones recogidas en los ítems.

\begin{tabular}{|ll|}
\hline $\begin{array}{l}\text { Totalmente en desacuerdo } \\
\text { Ni de acuerdo ni en desacuerdo }\end{array}$ & $\begin{array}{l}\text { En desacuerdo } \\
\text { De acuerdo }\end{array}$ \\
Totalmente de acuerdo & \\
\hline
\end{tabular}

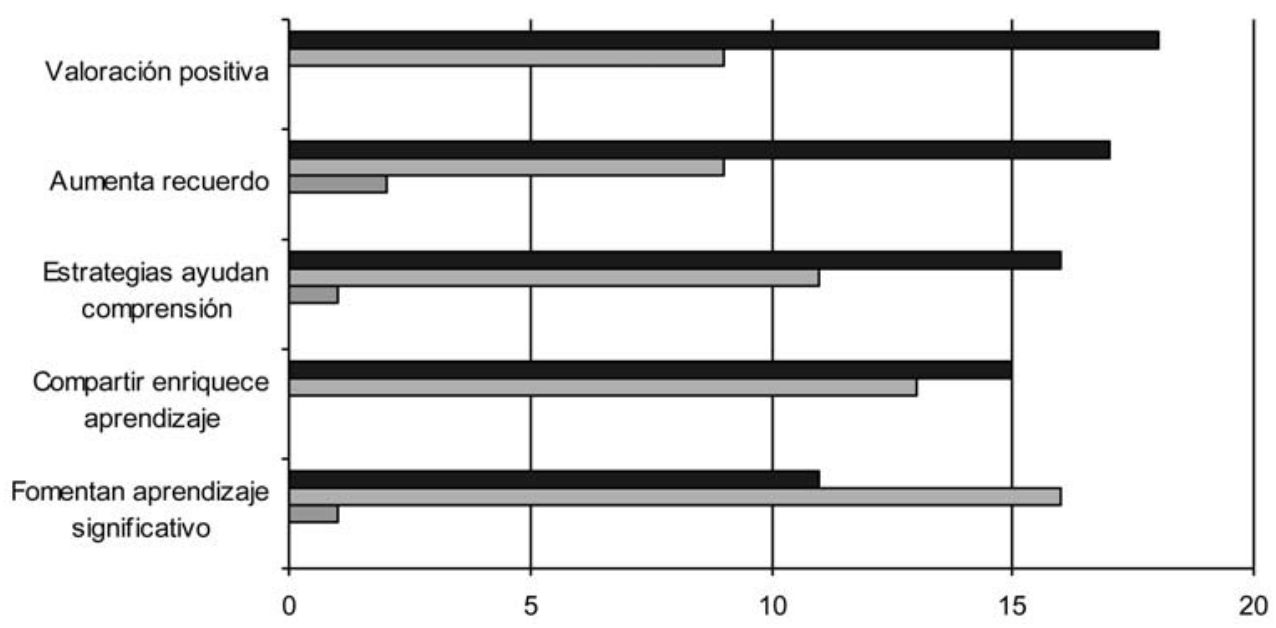

Figura 1. Frecuencias de respuesta a cada ítem de evaluación del aprendizaje

En concreto, para el $96.4 \%$ de los alumnos las estrategias utilizadas les han permitido alcanzar un aprendizaje más significativo que otro tipo de estrategias más receptivas, afirmando en un $100 \%$ que compartir propuestas con otras personas enriquece el aprendizaje. Por otro lado, para el $96.4 \%$ de los alumnos las estrategias como la elaboración de mapas conceptuales y la discusión grupal han ayudado a la comprensión del tema. El 92.8\% de los participantes indicaron que, aunque el aprendizaje autónomo y cooperativo conlleva más tiempo, facilita recordar a más largo plazo los contenidos aprendidos. Finalmente, el $100 \%$ de los alumnos valoraron positivamente la actividad.

\section{Ventajas e inconvenientes del aprendizaje autónomo y cooperativo}

Las ventajas de este tipo de aprendizaje, de acuerdo con la Figura 2, se agrupan en tres categorías: a) desarrollo de habilidades interpersonales; b) calidad del aprendizaje y, c) motivación y actitud positiva hacia el aprendizaje. 

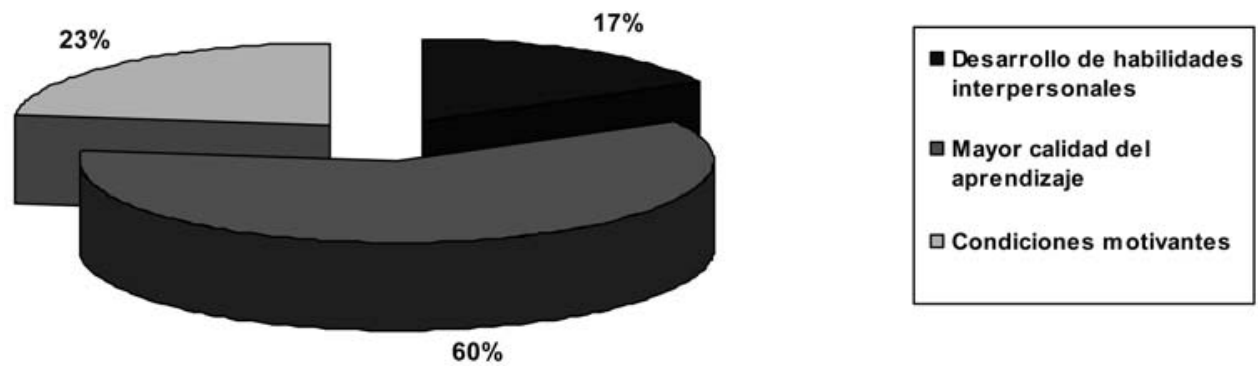

Figura 2. Porcentaje de tipos de ventajas percibidas

El $17 \%$ de las respuestas hacían referencia a ventajas en el desarrollo de habilidades interpersonales tales como la mejora de las competencias sociales, la resolución de conflictos que surgen en el grupo, las habilidades de trabajo en grupo y su cohesión. En un $60 \%$, las ventajas se relacionan con una mayor calidad del aprendizaje: enriquecimiento con las opiniones de los demás, mantenimiento del aprendizaje a más largo plazo y de manera más significativa, mayor profundización en los conocimientos, mejor comprensión y claridad de ideas, planteamiento de soluciones más reales y reflexión más crítica. Finalmente, el 23\% de las respuestas hacían referencia al resultado más motivador, la promoción de una actitud más positiva y una mayor implicación hacia el aprendizaje, en concreto, las clases resultan más amenas, activas y dinámicas, favoreciendo el esfuerzo hacia la lectura y permitiendo más autonomía en el aprendizaje.

Los resultados indicaron que entre estas ventajas, hay diferencias significativas a favor de la mayor calidad del aprendizaje respecto al fomento de habilidades interpersonales o motivacionales $\left(\chi^{2}(2)=21.16, p<.05\right)$.

Por otra parte, tal y como recoge la Figura 3, los inconvenientes se agrupan en tres categorías: a) inseguridad en el conocimiento adquirido, bien por su contenido o por no adquirir la totalidad de los contenidos $(34 \%)$; b) mayor tiempo de dedicación en comparación con la metodología de aprendizaje receptivo, destacando la imposibilidad de utilizarlo para el total de la asignatura $(36 \%)$ y, c) falta de habilidades interpersonales entre los componentes del grupo para poder realizar adecuadamente el trabajo en equipo (30\%), en concreto, la dificultad para llegar al consenso, la falta de cohesión o implicación.

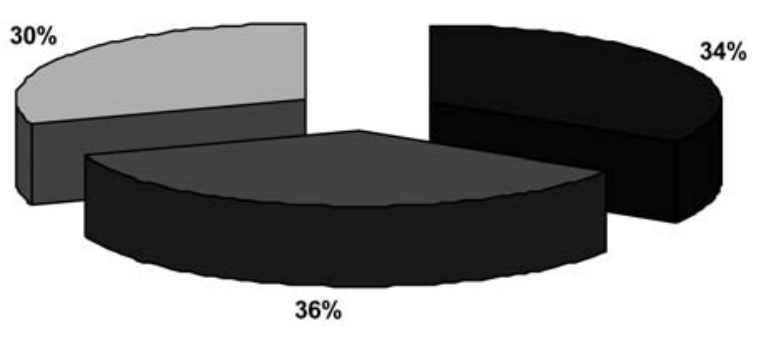

E Inseguridad en el conocimiento

口 Exige más tiem po

$\square$ Falta de habilidades interpersonales

Figura 3. Porcentaje de tipos de inconvenientes percibidos 
No existen diferencias significativas entre los inconvenientes hallados $\left(\chi^{2}(2)=\right.$ $.25, p>.05)$.

\section{Regulación de la planificación de las estrategias motivacionales}

Los resultados muestran que la mayoría de los participantes están Totalmente de acuerdo o De acuerdo con las afirmaciones presentadas, tal y como refleja la Figura 4.

La actividad parece haber facilitado para todos los alumnos que sean conscientes de que hay varias maneras de resolver un caso antes de adoptar una decisión final. Para un $92.9 \%$ y el $96.4 \%$ la actividad ha facilitado ser conscientes de la necesidad de planificar los recursos necesarios y de marcar objetivos antes de empezar a trabajar con los alumnos, respectivamente. Para un $89.3 \%$ de participantes la actividad les había ayudado a seleccionar cada estrategia motivacional con un propósito específico y plantearse el interés del alumno hacia el tema en su planificación previa (96.3\%). Finalmente, el $100 \%$ de los participantes considera que la actividad había fomentado un pensamiento divergente al enseñar un tema, adaptando las estrategias más adecuadas a las características del alumnado.

En suma, es positiva la valoración de la gestión metacognitiva que implica el proceso de enseñanza aprendizaje autónomo y cooperativo.

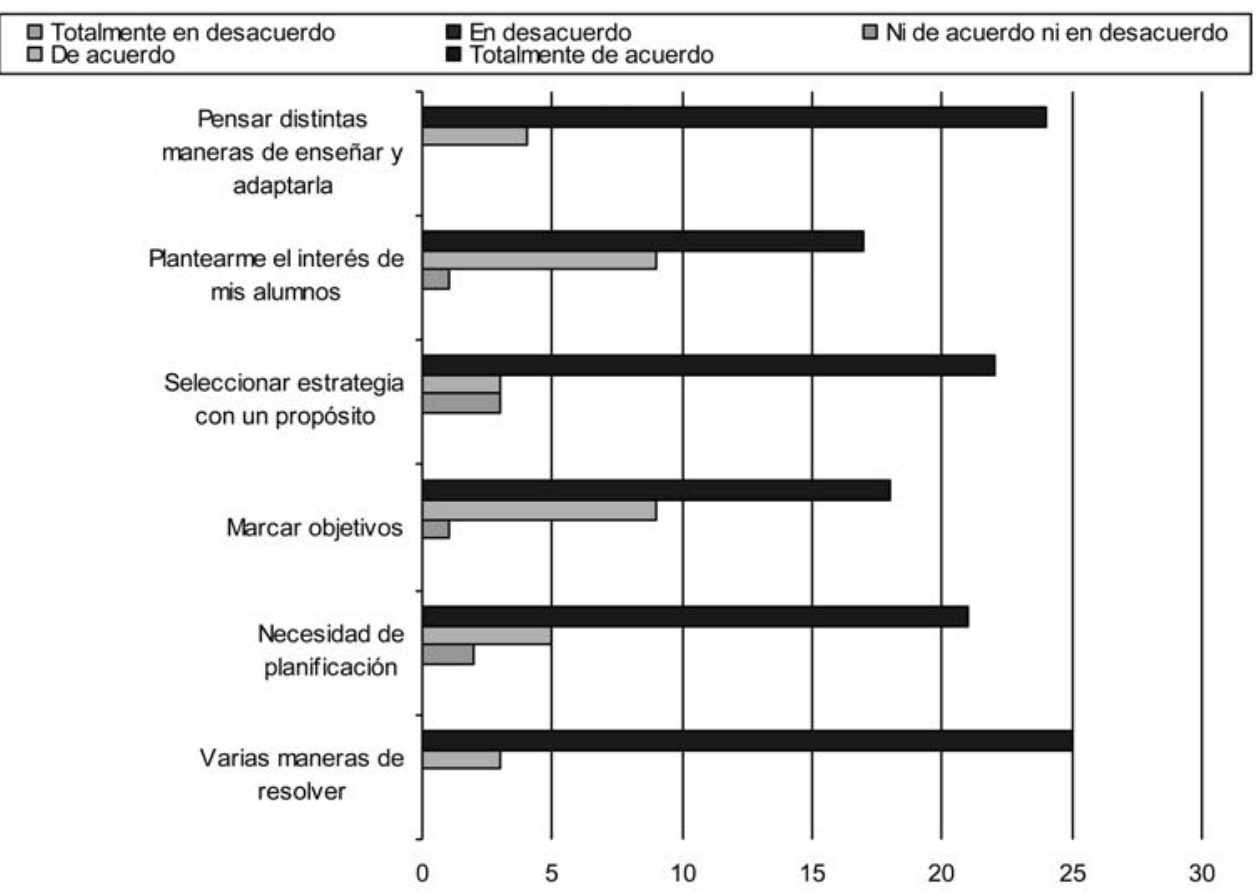

Figura 4. Frecuencias de respuesta a cada ítem de regulación de planificación de estrategias 


\section{DisCUSIÓN}

De la exposición de resultados, se desprende que la aplicación de un aprendizaje autónomo para la elaboración de un contenido de la asignatura de Psicología de la Educación combinado con el aprendizaje cooperativo tiene resultados positivos sobre la percepción de los alumnos universitarios acerca de su propio aprendizaje.

Estos resultados corroboran la importancia de potenciar la cooperación entre alumnos como una estrategia eficaz para promover la metacognición (Barkley et al., 2005; Pozo, 2008), especialmente cuando se combinan actividades de diversos formatos y distintos niveles de compromiso o interacción.

Sería interesante, a medio plazo, conocer la posible correlación existente entre la regulación metacognitiva de cada alumno y su calificación en la evaluación del tema trabajado.

Asimismo, en futuros trabajos sería interesante constatar empíricamente mediante la observación de la dinámica interactiva en los grupos cuáles son las competencias cooperativas como complemento de las respuestas al respecto. Dado que además la falta de éstas ha sido una de las dificultades mencionadas por los propios alumnos, su observación sistemática permitiría conocer fiablemente el tipo de carencias entre los alumnos que permitiera diseñar actividades y estrategias de optimización.

Es interesante resaltar que incitar a los estudiantes a reflexionar y ejercer un control metacognitivo sobre sus aprendizajes en distintos ámbitos de conocimiento y en diversas situaciones de aprendizaje facilita la generalización y transferencia del conocimiento. De igual manera, mejora el control metacognitivo a nuevos ámbitos y contextos de aprendizaje. En suma, se estará fomentando la gestión autónoma del aprendizaje (Pozo y Mateos, 2009).

En consecuencia, se sugiere que promover el aprendizaje entre universitarios puede facilitar que, progresivamente y de forma independiente, transfieran estrategias aprendidas a situaciones diferentes, cada vez más complejas y alejadas de las situaciones de aprendizaje originales (Mateos, 2001; Monereo y Pozo, 2003; Pozo, 2008).

Para finalizar, tal y como plantean Pozo y Mateos (2009) las nuevas demandas docentes no suponen tanto abandonar los viejos hábitos docentes sino repensarlos y restringir su función, completándolos con otras estrategias. Se trata de transferir de manera progresiva el control del aprendizaje al alumno. Siguiendo a Monereo, Pozo y Castelló (2001) existen diversas actividades y estrategias instruccionales que pueden utilizarse en este proceso y que varían en el nivel de autonomía del alumno. Desde estrategias de instrucción explícita como las instrucciones y el modelado, se puede avanzar con el análisis de casos y hojas de pensamiento hasta la discusión de procesos de pensamiento, la enseñanza cooperativa y recíproca. La tutoría entre iguales correspondería al ejemplo de práctica más autónoma.

Además, cada fase del proceso requiere una preparación concreta. Existen modelos que guían la puesta en marcha de este tipo de estrategias y la investigación sobre sus efectos. Como ya fue mencionado en la introducción, un ejemplo lo constituye el modelo DIDEPRO (De la Fuente y Justicia, 2007; De la Fuente et al., 2005). 
Asimismo, cada vez son más numerosas las publicaciones sobre aspectos concretos del proceso que deben tenerse en cuenta. Por nombrar uno, los materiales que se proporcionan a los alumnos y las actividades que se plantean, han recibido mucha atención. Hay autores (e.g., Argüelles, 2004-2005; Dickinson, 1987) que indican que deben cumplir ciertas condiciones: las actividades deben tener un diseño sencillo y ser suficientes y variadas para cubrir las diferentes necesidades de alumnos con estilos de aprendizaje diferentes y para mantener el interés. El lenguaje debe ser claro y adaptado al nivel de los alumnos. Se recomienda asimismo que las instrucciones se cuiden especialmente dado que son cruciales en este contexto y el papel de los ejemplos es destacable.

Por mencionar otros aspectos relevantes, se podrían destacar la retroalimentación y el clima. Ya no es suficiente con la calificación de las respuestas sino que deben acompañarse con explicaciones concretas sobre el porqué y el dónde de los posibles fallos para que se produzca un aprendizaje real (Pérez-Gómez, 2009). $\mathrm{Si}$, además, se consideran los numerosos factores que inciden en el desarrollo de un aprendizaje autónomo y sus competencias asociadas, por ejemplo, el clima del aula, un grado de confianza mutua, las expectativas (altas) del alumnado y profesorado, las interacciones positivas personales, aspectos emocionales, etc. conduce a revisar las habilidades docentes para reconfigurarlas en un nuevo marco de acción (Rué, 2009).

En cualquier caso, es necesario extender estas buenas prácticas de enseñanzaaprendizaje a otros contenidos y materias, convertirlas en la práctica habitual en el proceso de enseñanza-aprendizaje, y no reducirlas a experiencias aisladas como la del presente trabajo.

\section{REFERENCIAS BIBLIOGRÁFICAS}

ARGÜELLES ÁLVAREZ, I. (2004-2005). Adaptación de materiales y TICs para el fomento del aprendizaje autónomo. RESLA, 17-18, 19-33.

BARKLEY, E. F.; CROSS, K. P. y MAJOR, C. H. (2005). Collaborative learning techniques: A handbook for college faculty. San Francisco: Jossey-Bass.

BIGGS, J. (2001). Teaching for Quality Learning at University. (3ª ed.). Buckingham: Open University Press.

DE LA FUENTE, J. y JUSTICIA, F. (2003). Regulación de la enseñanza para la autorregulación del aprendizaje en la Universidad. Aula Abierta, 82, 161-171.

DE LA FUENTE, J. y JUSTICIA, F. (2007). The DIDEPRO® Model of Regulated Teaching and Self-Regulated Learning: Recent advances. Electronic Journal of Research in Educational Psychology, 5 (3), 535-564. Disponible en: http:// www.investigacionpsicopedagogica.org/revista/articulos/13/english/Art_13_209.pdf.

DE LA FUENTE, J.; JUSTICIA, F. y BERBÉN, A. B. G. (2005). An interactive model of regulated teaching and self-regulated learning. International Journal of Learning, 12, 217-225.

DE LA FUENTE, J.; PICHARDO, M. C.; JUSTICIA, F. y BERBÉN, A. (2008). Enfoques de aprendizaje, autorregulación y rendimiento en tres universidades europeas. Psicothema, 20, 705-711. 
DICKINSON, L. (1987). Supporting the learner in self-instruction. En L. Dickinson (Ed.), Self-instruction in Language Learning (pp. 88-105). Cambridge: Cambridge University Press.

ESCRIBANO, A. (1995). Aprendizaje cooperativo y autónomo en la enseñanza universitaria. Anuario Interuniversitario de didáctica, 13, 89-102.

GARCÍA-VALCÁRCEL, A. (1994). El proceso de enseñanza en las aulas universitarias. Una aproximación cualitativa. Qurriculum, 6-7, 155-174.

JOHNSON, D. W. y JOHNSON, R. T. (1989). Cooperative Learning: What Special Education Teachers Needs to Know. Pointer, 33 (2), 5-10.

JOHNSON, D. W.; JOHNSON, R. T. y HOLUBEC, E. (1999). El aprendizaje cooperativo en el aula. Barcelona: Paidós.

MATEOS, M. (2001). Metacognición y educación. Buenos Aires: Aique.

MATEOS, M. y PÉREZ, M. (2006). El cambio de las concepciones de los alumnos sobre el aprendizaje. En J. I. Pozo, N. Scheuer, M. P. Pérez Echeverría, M. Mateos, E. Martín y M. de la Cruz (Eds.), Nuevas formas de pensar la enseñanza y el aprendizaje. Las concepciones de profesores y alumnos (pp. 403-417). Barcelona: GRAÓ.

MONEREO, C. y POZO, J. I. (2003). La cultura educativa en la Universidad: Nuevos retos para profesores y alumnos. En C. Monereo y J. I. Pozo (Eds.), La Universidad ante la nueva cultura educativa. Enseñar y aprender para la autonomía (pp. 15-29). Madrid: Síntesis.

PÉREZ-GÓMEZ, A. (2009). La evaluación como aprendizaje. Córdoba: Universidad de Córdoba.

POZO, J. I. (2003). Adquisición de conocimiento: cuando la carne se hace verbo. Madrid: Morata.

POZO, J. I. (2008). Aprendices y maestros: la psicología cognitiva del aprendizaje. Madrid: Alianza.

POZO, J. I. y MATEOS, M. (2009). Aprender a aprender: hacia una gestión autónoma y metacognitiva del aprendizaje. En J. I. Pozo y M. P. Pérez Echeverría (Coords.), Psicología del aprendizaje universitario: La formación en competencias (pp. 54-69). Madrid: Ediciones Morata.

POZO, J. I. y MONEREO, J. I. (2009). Introducción: La nueva cultura del aprendizaje universitario o por qué cambiar nuestras formas de enseñar y aprender. En J. I. Pozo y M. P. Pérez Echeverría (Coords.), Psicología del aprendizaje universitario: La formación en competencias (pp. 9-28). Madrid: Morata.

POZO, J. I.; MONEREO, C. y CASTELLÓ, M. (2001). El uso estratégico del conocimiento. En C. Coll, J. Palacios y A. Marchesi (Coords.), Psicología de la Educación Escolar (pp. 211-233). Madrid: Alianza Editorial.

ROSÁRIO, P. (2004). Estudar o Estudar: As (Des)venturas do Testas. Porto: Porto Editora.

RUÉ, J. (2009). El aprendizaje autónomo en educación superior. Madrid: Narcea. 
SAMPASCUAL, G. (2007). La motivación. En G. Sampascual, Psicología de la educación (pp. 284-313). Madrid: UNED.

SCHRAW, G. y DENNISON, R. S. (1994). Assessing metacognitive awareness. Contemporary Educational Psychology, 19, 460-475.

STATISTICAL PACKAGE FOR THE SOCIAL SCIENCES. (2006). SPSS Base 15.0 User's Guide. Chicago, IL: SPSS Inc.

WEINERT, E. y KLUWE, R. (1987). Metacognition, Motivation and Understanding. Hillsondale: Lawrence Erlbaum Associates, Publishers.

ZIMMERMAN, B. J. y KITSANTAS, A. (1997). Developmental phases in selfregulation: Shifting from process goals to outcome goals. Journal of Educational Psychology, 82, 51-59.

\section{ANeXos}

\section{Anexo 1: Caso práctico propuesto}

Imagina que tus alumnos de $4^{\circ}$ han participado en un estudio recientemente. Los resultados de dicho estudio indican que sus intereses para aprender inglés son muy dispares y que presentan diversas quejas en referencia a cómo se les enseña hasta el momento. Entre las quejas que presentan, hay comentarios de diferentes niños como los que siguen:

- "a mi me gustaría entender una canción en inglés pero lo que hacemos en clase no sirve para esto..."

- "yo para el inglés no valgo, veo que otros compañeros son más hábiles y a veces hay alumnos que se ríen de mi..."

- "no tengo ni idea de inglés y el nivel es demasiado alto en clase, cada vez que tengo que responder a una pregunta me siento muy angustiado y me bloqueo..."

- "en inglés me aburro porque solo hacemos verbos..."

- "yo ser de Ucrania y hablar mejor inglés que español, clase inglés mucho fácil...yo aburrir..."

- "por más que estudie, nunca conseguiré aprender ese vocabulario ni decir esos sonidos tan complicados..."

- "no entiendo para qué tanto inglés, si yo nunca viajo..."

- "yo voy desde los seis años a clases particulares y en clase se dan cosas muy básicas...".

Como buen maestro que eres, te planteas estudiar la situación y plantear una estrategia para que todos los niños se sientan motivados en tu clase. Para ello, vas a estudiar tres teorías básicas sobre motivación y vas a buscar estrategias para fomentarla. 


\section{Anexo 2: Cuestionario elaborado ad hoc}

\section{EVALUACIÓN METACOGNITIVA DE LA ACTIVIDAD}

A continuación se presentan una serie de afirmaciones sobre la forma en la que has estudiado el tema de Motivación. Por favor, señala la opción que mejor describa tu postura:

Estoy:

\begin{tabular}{|l|l|l|l|l|l|}
\hline Esta forma de estudiar el tema: & $\begin{array}{c}\text { Totalmente } \\
\text { de acuerdo }\end{array}$ & $\begin{array}{c}\text { De } \\
\text { acuerdo }\end{array}$ & $\begin{array}{c}\text { Ni de } \\
\text { acuerdo } \\
\text { ni en } \\
\text { desacuerdo }\end{array}$ & $\begin{array}{c}\text { En } \\
\text { desacuerdo }\end{array}$ & $\begin{array}{c}\text { Totalmente } \\
\text { en } \\
\text { desacuerdo }\end{array}$ \\
\hline $\begin{array}{l}\text { 1. Me ha permitido alcanzar un aprendizaje } \\
\text { más significativo que otro tipo de } \\
\text { estrategias más receptivas. }\end{array}$ & & & & & \\
\hline $\begin{array}{l}\text { 2. Me ha permitido constatar que compartir } \\
\text { mis propuestas con otras personas } \\
\text { enriquece mi aprendizaje. }\end{array}$ & & & & & \\
\hline $\begin{array}{l}\text { 3. Estrategias como la elaboración de mapas } \\
\text { conceptuales y la discusión grupal me han } \\
\text { ayudado a la comprensión del tema. }\end{array}$ & & & & & \\
\hline $\begin{array}{l}\text { 4. Aunque conlleva más tiempo, creo que } \\
\text { recordaré a largo plazo los contenidos } \\
\text { aprendidos. }\end{array}$ & & & & & \\
\hline 5. La valoro positivamente. & & & & & \\
\hline
\end{tabular}

A continuación, señala tres puntos fuertes de este tipo de aprendizaje:

1.

2 .

3.

Asimismo, plantea tres críticas a este tipo de aprendizaje:

4.

5.

6.

Finalmente, se presentan una serie de afirmaciones sobre la actividad realizada. Por favor, señala la opción que mejor describa tu postura:

\begin{tabular}{|l|l|l|l|l|l|}
\hline Esta actividad me ha ayudado a: & Sí & $\begin{array}{c}\text { Más } \\
\text { bien sí }\end{array}$ & $\begin{array}{c}\text { Postura } \\
\text { intermedia }\end{array}$ & $\begin{array}{c}\text { Más } \\
\text { bien no }\end{array}$ & No \\
\hline $\begin{array}{l}\text { 1. Ser consciente de que hay varias maneras de resolver un mismo } \\
\text { caso antes de adoptar una decisión final. }\end{array}$ & & & & \\
\hline $\begin{array}{l}\text { 2. Ver la necesidad de planificar los recursos que necesitaré para } \\
\text { resolver un problema motivacional en mis alumnos/as. }\end{array}$ & & & & & \\
\hline 3. Marcar objetivos antes de empezar a trabajar con mis alumnos/as. & & & & & \\
\hline $\begin{array}{l}\text { 4. Seleccionar cada estrategia motivacional con un propósito } \\
\text { específico. }\end{array}$ & & & & & \\
\hline $\begin{array}{l}\text { 5. Plantearme el interés que tendrán mis alumnos/as cada vez que } \\
\text { inicie un tema. }\end{array}$ & & & & & \\
\hline $\begin{array}{l}\text { 6. Pensar en distintas maneras de enseñar un tema y valorar cuál se } \\
\text { adapta más a las características de mis alumnos/as. }\end{array}$ & & & & & \\
\hline
\end{tabular}

